\title{
First order phase transition of a long polymer chain
}

\author{
David Aristoff and Charles Radin \\ Mathematics Department, University of Texas, Austin, TX 78712, USA \\ E-mail: radin@math.utexas.edu
}

Received 15 November 2010, in final form 16 December 2010

Published 17 January 2011

Online at stacks.iop.org/JPhysA/44/065004

\begin{abstract}
We consider a model consisting of a self-avoiding polygon occupying a variable density of the sites of a square lattice. A fixed energy is associated with each $90^{\circ}$ bend of the polygon. We use a grand canonical ensemble, introducing parameters $\mu$ and $\beta$ to control average density and average (total) energy of the polygon, and show by Monte Carlo simulation that the model has a first order, nematic phase transition across a curve in the $\beta-\mu$ plane.
\end{abstract}

PACS numbers: 82.35.Lr, 64.70.M-, 64.60.De, 36.20.Fz

(Some figures in this article are in colour only in the electronic version)

\section{Introduction}

In polymer physics self-avoiding walks have been used for many years to model long chain molecules [1, 2]. One of the oldest such models, due to Flory [3], consists of a single selfavoiding random polygon occupying all the sites of a square lattice, with the randomness controlled by the total energy associated with $90^{\circ}$ bends in the polygon. Thinking of the polygon as made of many flexibly connected monomers, the model is a canonical ensemble with the temperature behavior analyzed only at the optimally high particle density of 1 . At high temperature the Flory model behaves like a disordered fluid, while at low temperature the model displays long-range nematic order. (Flory was modeling the 'melting' of a nematically ordered polymer [3].) Although it is generally accepted that there is a true phase transition between these regimes in the model, there has been a dispute over the character of the transition [4], which Flory had originally predicted to be first order. For the Flory model, the controversy seems to have been settled by a recent paper [5] of Jacobsen and Kondev which shows that the transition is second order. (There is a useful summary of the history of the controversy in [5].) They also suggest, however, that the transition may become first order at lower density if vacancies are permitted (see section VII C in [5]). In our paper, we concentrate on this generalization of the Flory model which allows for vacant lattice sites, so that our random polygon is now controlled by two parameters, temperature and chemical potential. We show by Monte Carlo simulation that the model has a first order phase transition. We also use order parameters to illustrate the nematic nature of the transition. 

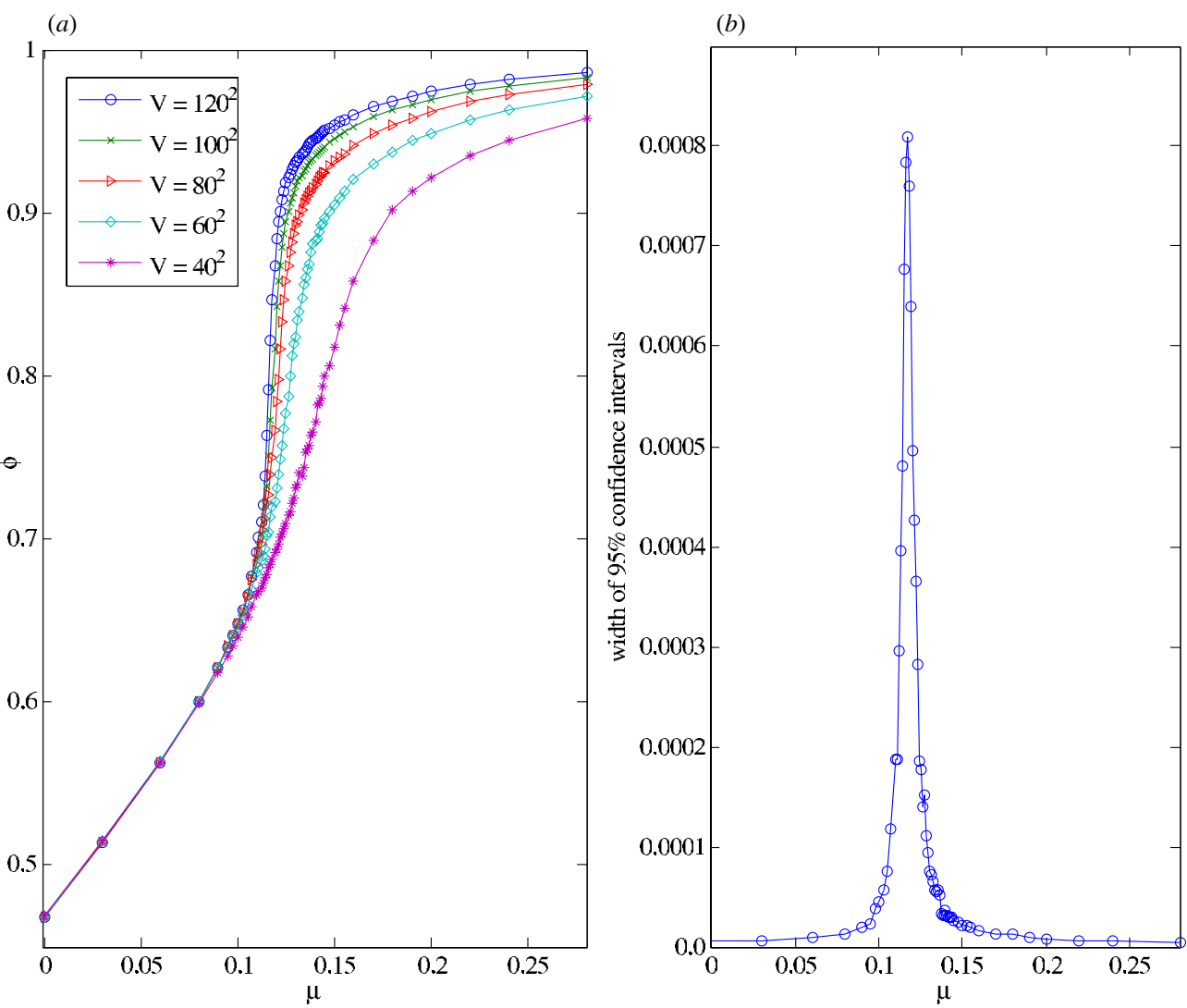

Figure 1. (a) The graph of average density versus $\mu$ at $\beta=1.5$, for system volumes $V=40^{2}$ through $V=120^{2}$. (b) Width of $95 \%$ confidence intervals for average density, for $V=120^{2}$.

We note some other variations and applications of the Flory model. One variation uses an ensemble of polygons rather than one long polygon. Although the connection between the two models is unclear, it is of interest that there have been conflicting results on the character of the transition in this case also [6, 7]. In a different direction, we note that variants of the Flory model have been applied to nonequilibrium materials, specifically to crumpled or confined sheets (in three dimensions) and wires (in two or three dimensions). For instance in [8], we modeled a progressively confined wire in two dimensions by a version of the model used here, but on a triangular lattice, showing a similar transition. A field theoretic (continuum) model of a variably confined wire by Boué and Katzav [9], however, found a second order transition, and it remains to understand the origins of this difference between their continuum model and our lattice models.

\section{The model and results}

Consider the set $\mathcal{W}$ of all self-avoiding polygons (i.e., closed self-avoiding loops) on the square lattice with periodic boundary conditions, $L=(\mathbb{Z} / v \mathbb{Z})^{2}$, with $v$ a fixed positive integer. The energy $E(w)$ of polygon $w$ in $\mathcal{W}$ is defined as the number of right angles in $w$, and the length $N(w)$ of $w$ is the number of unit line segments in $w$. Given an inverse temperature 
(a)

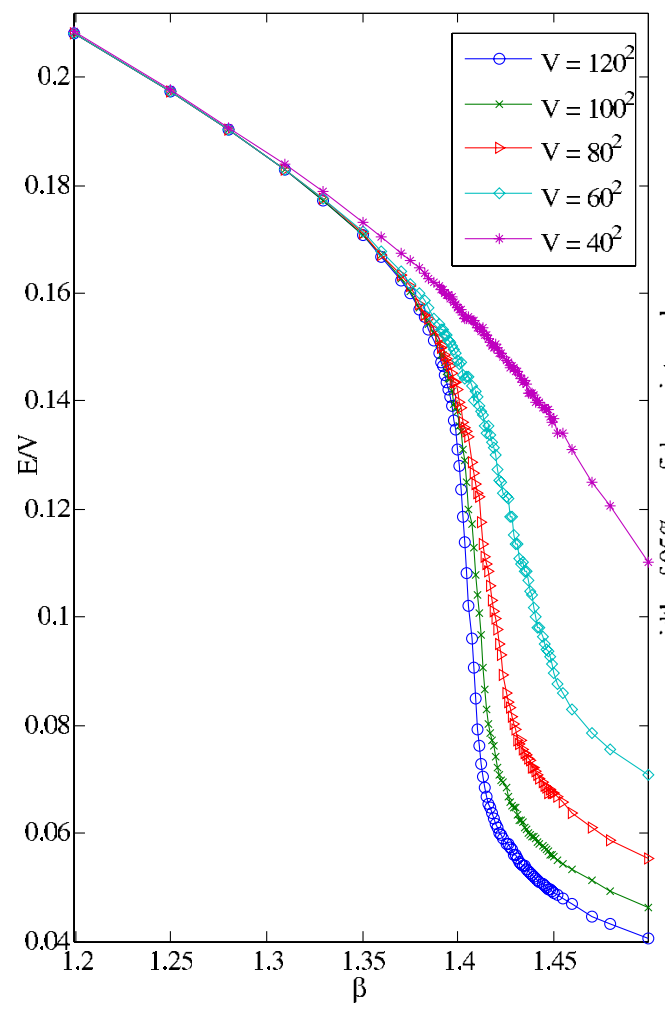

(b)

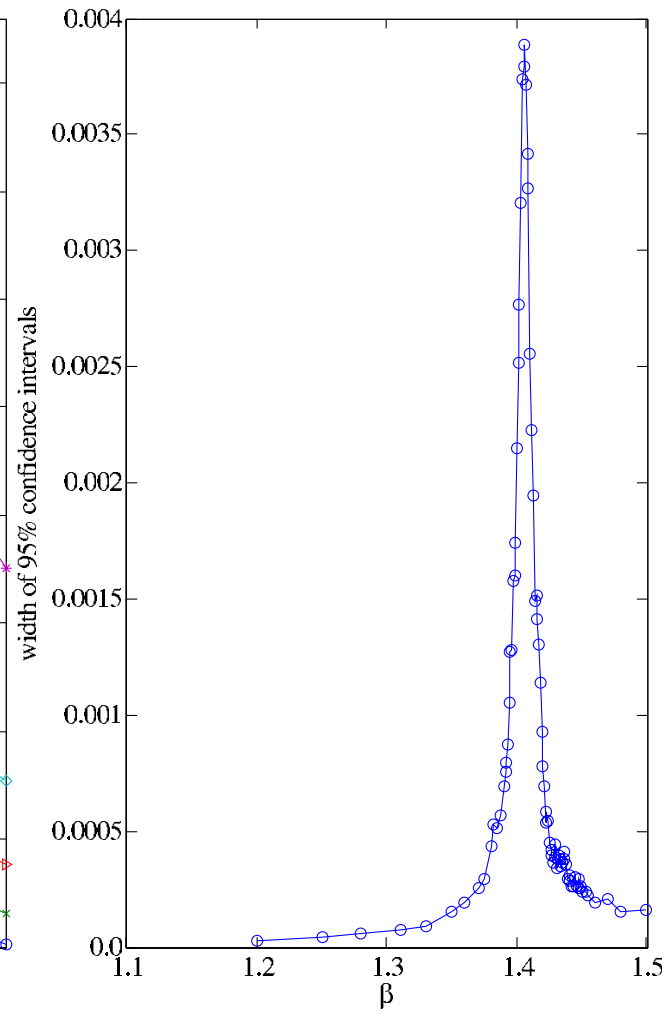

Figure 2. (a) The graph of average energy per volume versus $\beta$ at $\mu=0.15$, for system volumes $V=40^{2}$ through $V=120^{2}$. (b) Width of $95 \%$ confidence intervals for average energy per volume, for $V=120^{2}$.

$\beta=1 / T$ and a chemical potential $\mu$, the free energy of the model is $\beta E-\beta \mu N-S(E, N)$, where $S(E, N)$ is the entropy, that is, the natural logarithm of the volume in phase space of self-avoiding polygons at fixed $E$ and $N$.

As usual in a grand canonical ensemble, this is optimized by the probability measure $m_{\beta, \mu}$ defined on the subsets of $\mathcal{W}$ by

$$
m_{\beta, \mu}(w)=\frac{1}{Z_{\beta, \mu}} \mathrm{e}^{-\beta(E(w)-\mu N(w))},
$$

for $w \in \mathcal{W}$, where $Z_{\beta, \mu}$ is the appropriate normalization. In this notation, we have suppressed the dependence of $m_{\beta, \mu}$ and $Z_{\beta, \mu}$ on the system volume $V=v^{2}$.

To simulate the model, we fix either $\beta$ or $\mu$, and then slowly increase the other parameter, starting from well into the disordered regime. The basic Monte Carlo step is as follows (see pp 41-4 in [10]). Given a polygon $w(t)$ at step $t$ in the simulation, we introduce, with probability $p_{i}$, a trial configuration $w(t)^{\prime}$ which changes the length of $w(t)$ by $\ell_{i}$. If $w(t)^{\prime}$ is not self-avoiding, then we take $w(t+1)=w(t)$; otherwise, we set $w(t+1)=w(t)^{\prime}$ with probability $q=\min (Q, 1)$, and $w(t+1)=w(t)$ with probability $1-q$, where

$$
Q=\mathrm{e}^{\beta\left[\mu \ell_{i}+E(w(t))-E\left(w(t)^{\prime}\right)\right]} .
$$


(a)

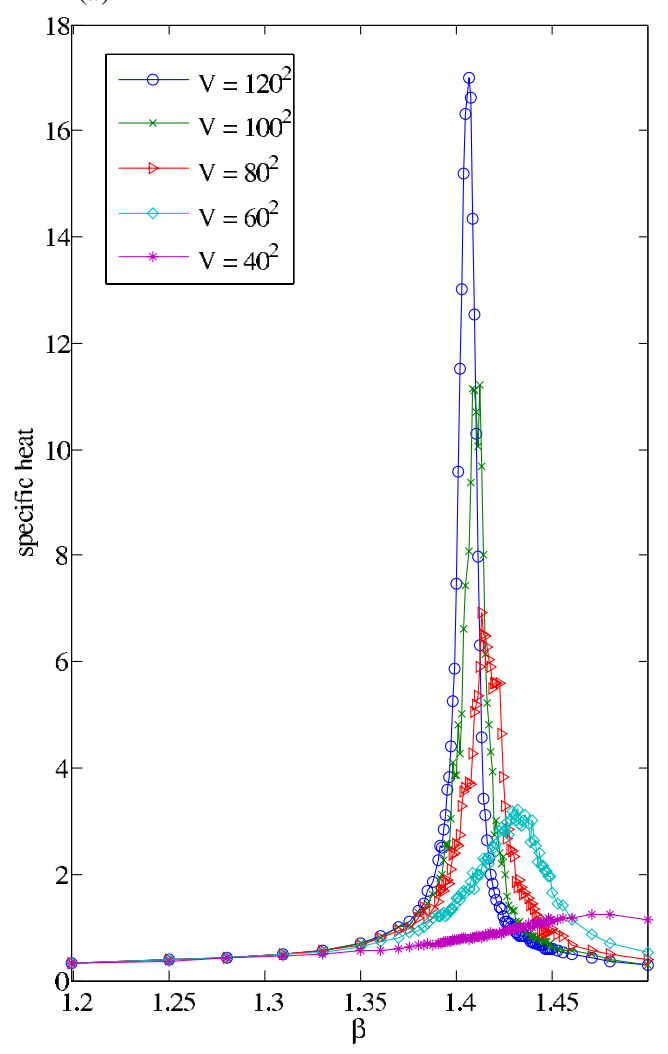

(b)

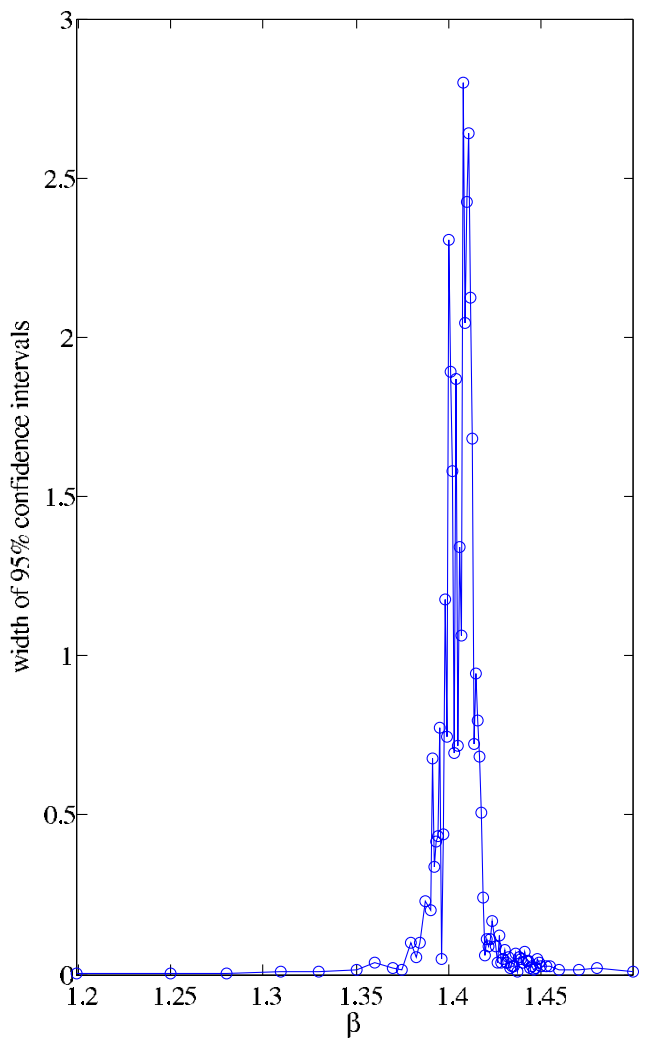

Figure 3. (a) The graph of specific heat versus $\beta$ at $\mu=0.15$, for system volumes $V=40^{2}$ through $V=120^{2}$. (b) Width of $95 \%$ confidence intervals for specific heat, for $V=120^{2}$.

Here, $p_{1}=p_{2}=2 / 5, p_{3}=1 / 5, \ell_{1}=-2, \ell_{2}=2$ and $\ell_{3}=0$. (The choices of the $p_{j}$ were somewhat arbitrary.)

To determine whether the simulation for each pair $(\beta, \mu)$ has sufficiently many Monte Carlo steps, we compute a 'mixing time' as the smallest $t$ such that the standard autocorrelation function

$$
\frac{1}{(n-t) \sigma^{2}} \sum_{i=1}^{n-t}\left(\operatorname{meas}\left(w_{i}\right)-\lambda\right) \cdot\left(\operatorname{meas}\left(w_{i+t}\right)-\lambda\right)
$$

falls below zero. Here, 'meas' represents any of our various measurements, described below, $\lambda$ and $\sigma^{2}$ are the sample average and variance (respectively) of meas over the simulation of $(\beta, \mu)$, and $w_{i}$ is the $i$ th configuration in the simulation of $(\beta, \mu)$, with $n$ total steps. We found that our simulations of each $(\beta, \mu)$ were, in the worst cases, at least five mixing times long (on average), and we therefore believe our Monte Carlo runs are reasonably close to sampling the distributions $m_{\beta, \mu}$. (For $\mu$ between -0.05 and 0.06, mixing times, in units of Monte Carlo steps, are between $2 \times 10^{7}$ and $4 \times 10^{7}$, while mixing times increase to about $2 \times 10^{9}$ near the transition $\mu$.)

We repeated each of our simulations 100 times, and obtained $95 \%$ confidence intervals for meas from Student's $t$-distribution with 99 degrees of freedom on the average values of meas 


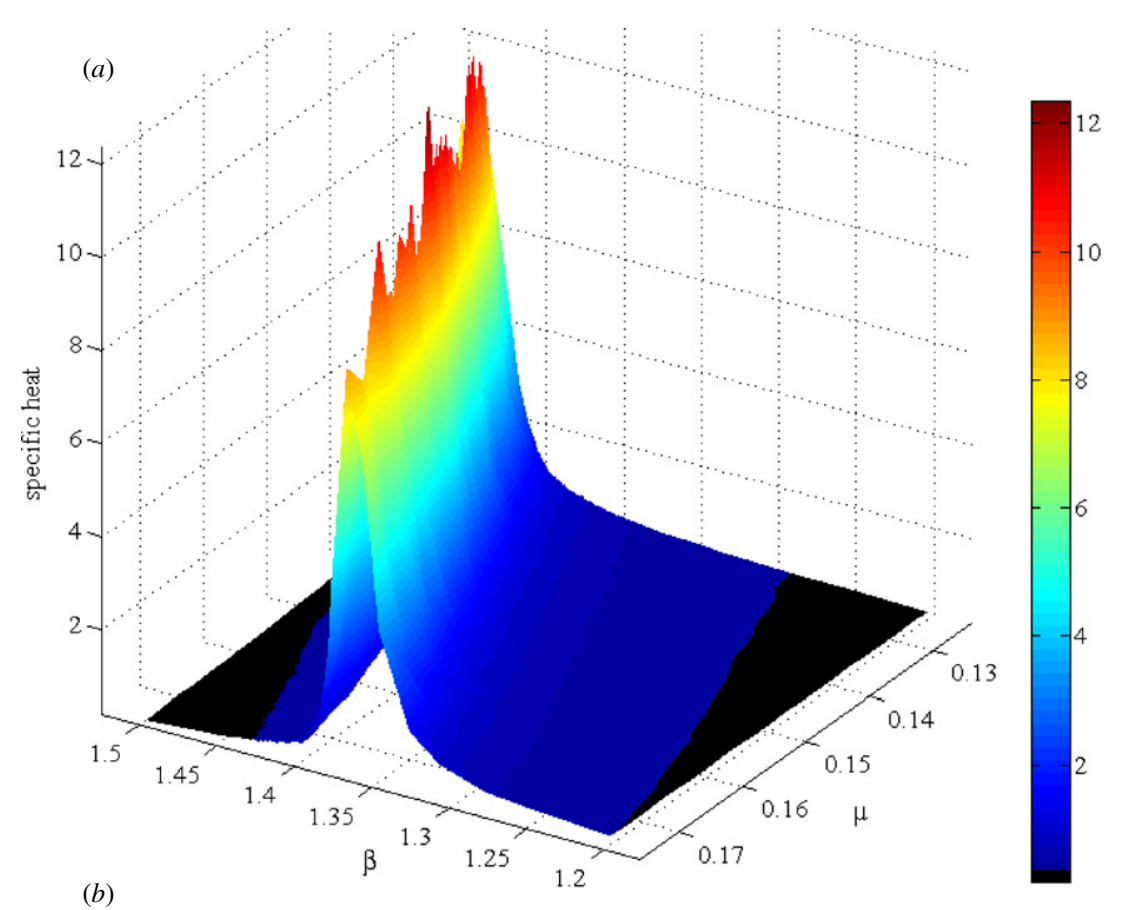

(b)

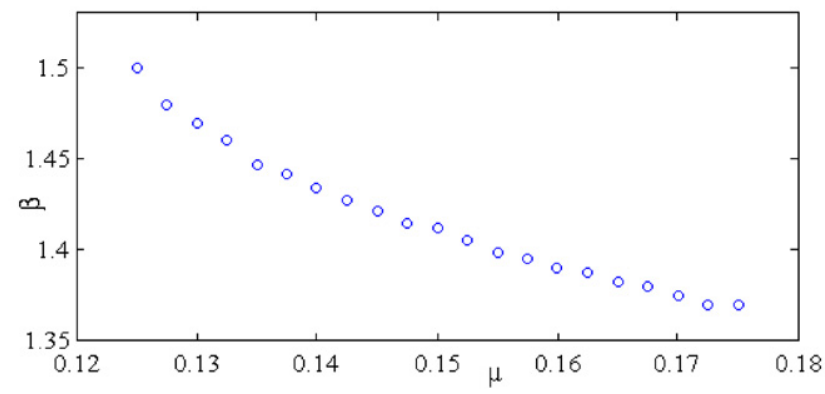

Figure 4. (a) The graph of specific heat versus $\beta$ and $\mu$, for $V=100^{2}$. (b) Estimation of $\beta$ values which maximize specific heat at fixed $\mu$.

over each simulation. (Measurements related to specific heat were calculated differently, and are discussed below.) A single simulation of the largest system $\left(V=120^{2}\right)$ contains $8 \times 10^{11}$ basic Monte Carlo steps, with $10^{10}$ Monte Carlo steps in the simulation of each of 80 points $(\beta, \mu)$.

We measure average energy per volume $\langle E\rangle_{\beta, \mu} / V$, average density $\langle\phi\rangle_{\beta, \mu}$, as well as order parameters 'corr' and 'lay', which were introduced in [8] and are defined as follows.

Given a polygon $w, \operatorname{corr}(w)$ is the proportion of edges in $w$ which have the same orientation (horizontal or vertical) as a randomly chosen edge in $w$. Given $w$, lay $(w)$ is the normalized volume $u^{2} / V$ of the largest square sublattice $L^{\prime}=(\mathbb{Z} / u \mathbb{Z})^{2} \subset L$ such that the orientation of $w$ (horizontal or vertical) at the origin agrees with the orientation of $w$ at $80 \%$ or more of the sites in $L^{\prime}$. (We choose an orientation for the polygon $w$ so that each lattice site has a unique horizontal or vertical orientation.) 
(a)

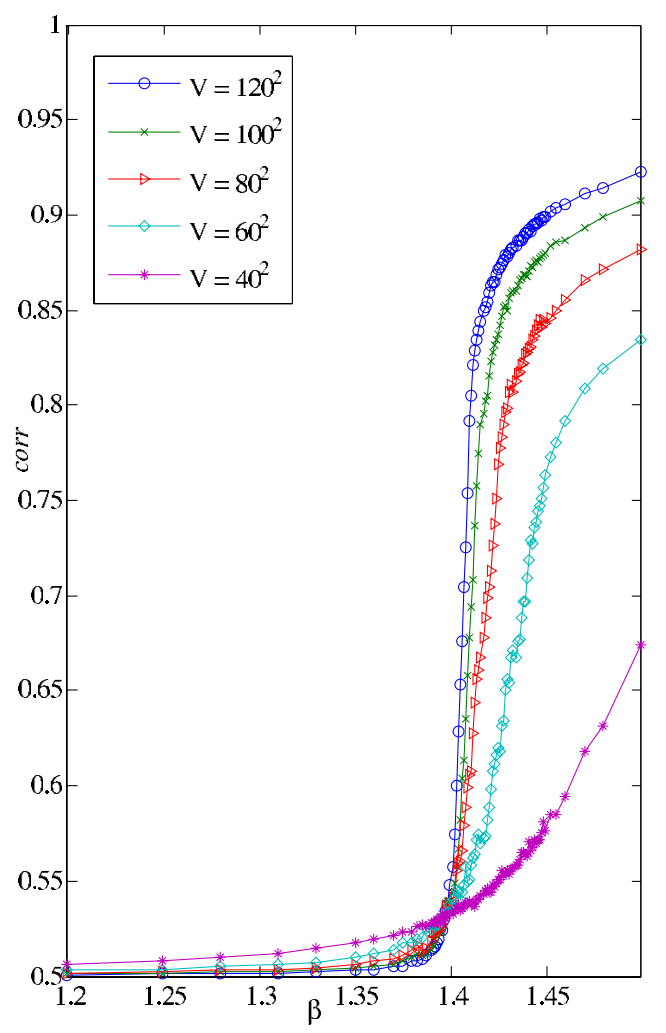

(b)

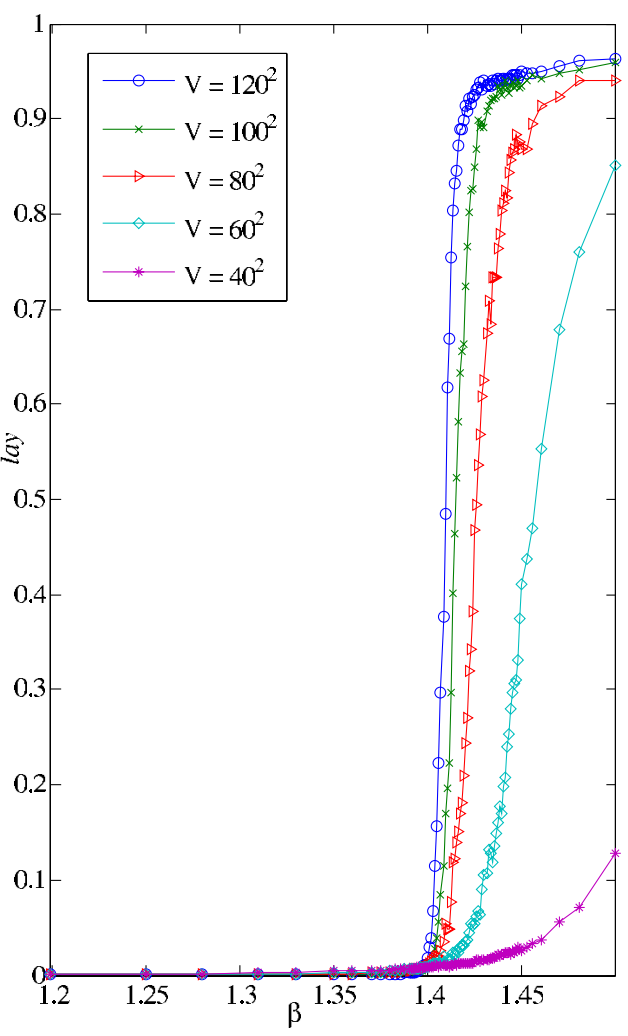

Figure 5. (a) The graph of corr versus $\beta$ at $\mu=0.15$, for volumes $V=40^{2}$ to $V=120^{2}$. (b) The graph of lay versus $\beta$ at $\mu=0.15$, for volumes $V=40^{2}$ to $V=120^{2}$.

We compute the specific heat $(1 / V) \partial\langle E\rangle_{\beta, \mu} / \partial T$ from fluctuations, that is,

$$
T^{2} \frac{\partial\langle E\rangle_{\beta, \mu}}{\partial T}=\langle E\rangle_{\beta, \mu}\langle\mu N-E\rangle_{\beta, \mu}-\left\langle E \mu N-E^{2}\right\rangle_{\beta, \mu} .
$$

To compute the values of $\langle\cdot\rangle_{\beta, \mu}$ from equation (4), we took averages of the relevant measurements meas over 100 independent simulations. Then, for $95 \%$ confidence intervals we repeated this process four times, and used Student's $t$-distribution with three degrees of freedom. We checked that the resulting curve agreed with the numerical derivative of energy.

In contrast with [8], we simulate well into the ordered regime and find direct evidence of a first order phase transition. In particular, the trends with increasing system size in the curves of figures 1 and 2 strongly suggest that the average density and average energy per volume both develop jump discontinuities at the transition, in the infinite volume limit. In confirmation, figure 3 shows the specific heat developing a delta function singularity at the transition.

We plot the specific heat surface as a function of $\beta$ and $\mu$, as well as the $(\beta, \mu)$ coordinates of the maximum of specific heat, at various $0.125 \leqslant \mu \leqslant 0.175$ and $1.2 \leqslant \beta \leqslant 1.5$ in figure 4 . The latter gives an indication of the transition curve; note that as $\mu$ increases, the temperature at which the transition occurs increases. 
(a)

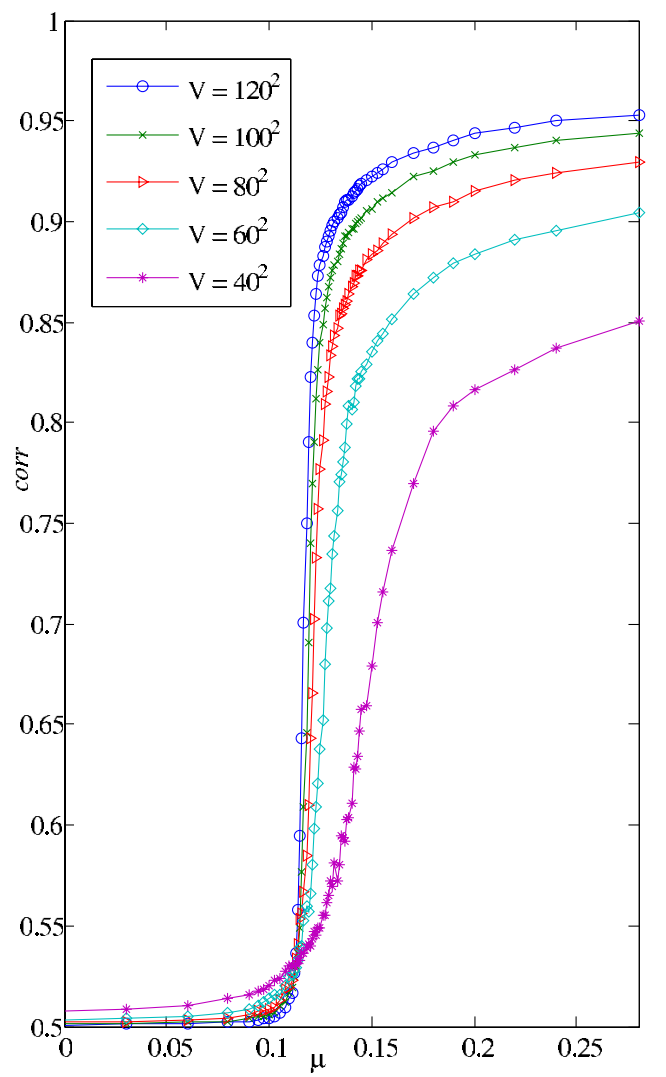

(b)

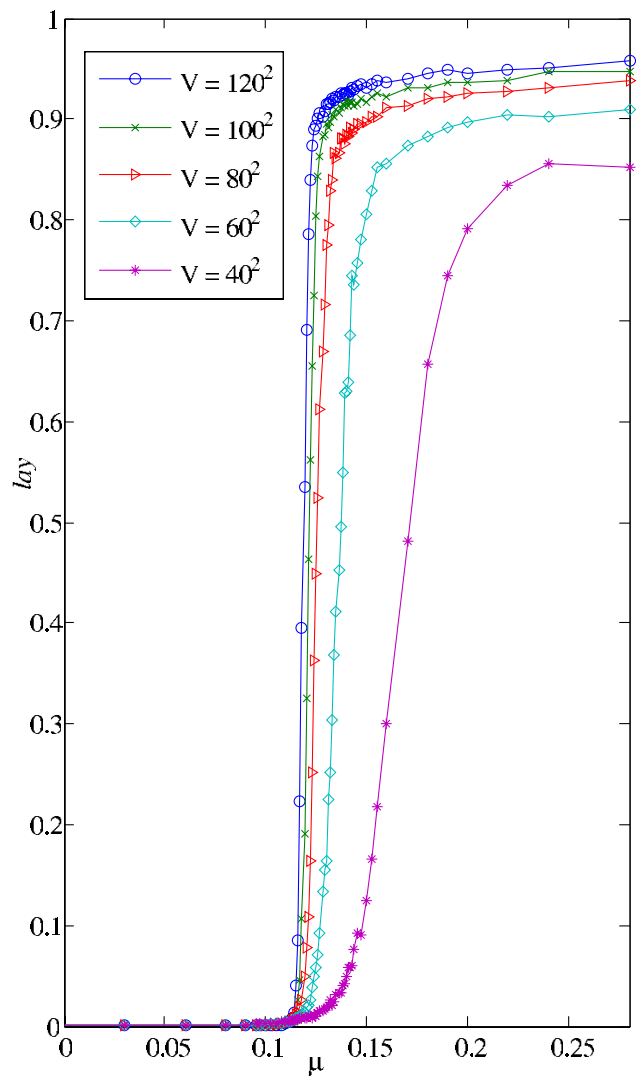

Figure 6. (a) The graph of corr versus $\mu$ at $\beta=1.5$, for volumes $V=40^{2}$ to $V=120^{2}$. (b) The graph of lay versus $\mu$ at $\beta=1.5$, for volumes $V=40^{2}$ to $V=120^{2}$.

As evidence of a nematic transition, the measurements corr and lay (see figures 5 and 6) exhibit a jump discontinuity at the transition from their disorder values of $1 / 2$ and zero, respectively.

Our Monte Carlo simulations incorporate many moves which are 'rejected'. In fact the rejection rate ranges from about $95 \%$ to almost $99 \%$, depending on $(\beta, \mu)$, with higher rejection rates at large $\beta$ or $\mu$. Simulations of a similar model using a rejectionless algorithm have been published [11, 12]. The rejectionless algorithm works only for random strings (with ends) rather than our random loops, but is likely faster than the algorithm we used for the system volumes $V$ we consider here.

\section{Nonequilibrium}

We mentioned above that with variable density added, a version of the Flory model on a triangular lattice has been used [8] to model wires progressively confined in two dimensions. (See [13] for a related approach, and further references.) We add here a note on the modeling of such nonequilibrium materials. Since the polygons are self-avoiding, these lattice models introduce a unit length scale for the width of the wire. Now if it requires energy $E$ to bend 
(a)

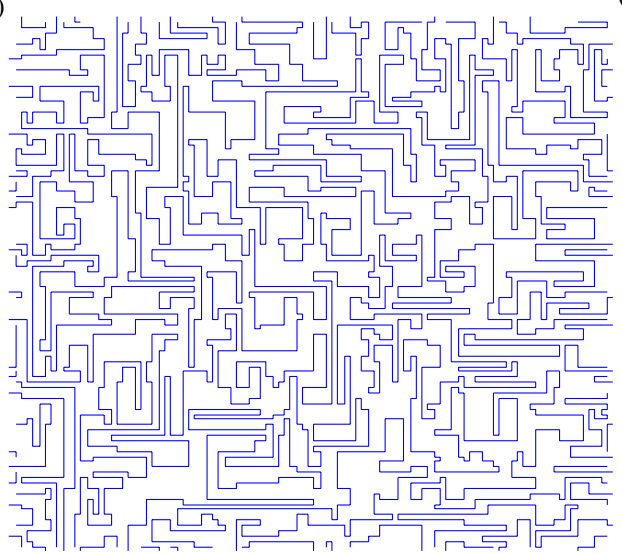

(c)

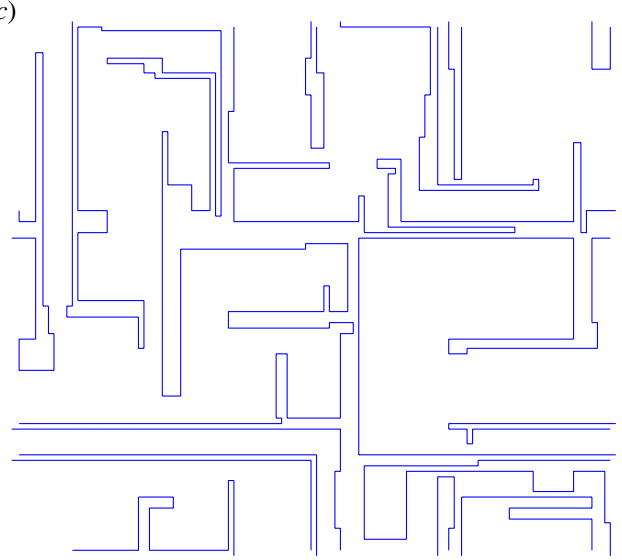

(b)

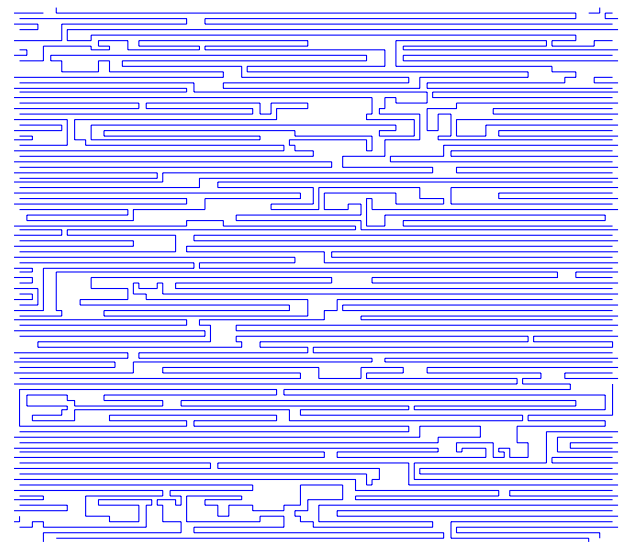

$(d)$

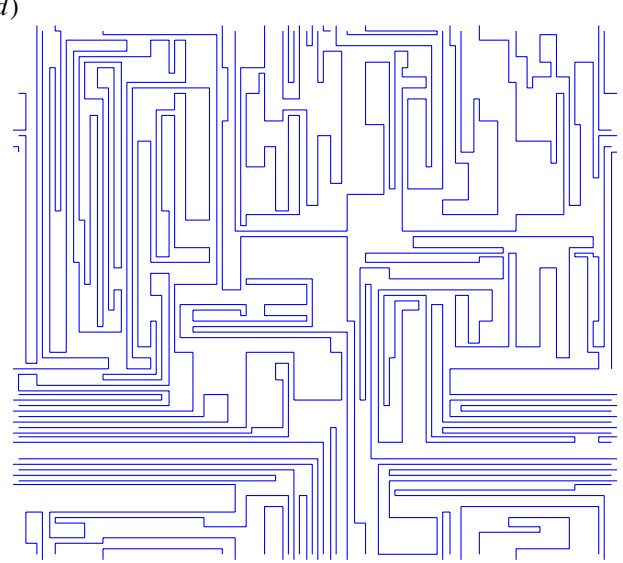

Figure 7. Configurations with $V=100^{2}$ in equilibrium at: $(a)(\beta, \mu)=(1.5,0.03)$ and density $\phi=0.515,(b)(\beta, \mu)=(1.5,0.124)$ and density $\phi=0.894,(c)(\beta, \mu)=(2.5,0.01)$ and density $\phi=0.146$, and $(d)(\beta, \mu)=(2.5,0.04)$ and density $\phi=0.529$.

one wire of unit thickness to a given radius of curvature, it would require $m E$ to bend a loose bundle of $m$ parallel wires, but because of the interconnectedness it would require more than $m E$ to bend a single wire of thickness $m$. Therefore, the bending energy is highly nonlinear in the thickness of the wire, growing faster than the square of the thickness, and so energy is an independent parameter in our modeling. Figure 7 shows typical polygons with low and high volume fraction, but at two very different temperatures. This could be useful in estimating whether experimentally confined materials are in an ordered or disordered regime. An alternative method would be to compute order parameters such as lay or corr.

\section{Conclusion}

We have introduced a version of the Flory model that allows for a positive fraction of vacancies, and shown by Monte Carlo simulation that the model has a first order, nematic phase transition. In terms of the (inverse) temperature $\beta$ and chemical potential $\mu$ of our grand canonical ensemble, we find that the transition lies approximately on the curve shown in figure 4(b). 
We conclude by contrasting the results in this paper with those in [8], which uses a very similar model, but on a triangular lattice and therefore with greater complexity of the interaction energies. A grand canonical ensemble was also used in [8], but simulations were confined to a single isotherm.

The evidence in [8] of a nematic transition is based on two order parameters, lay and corr, used also in this paper. The arguments in [8] are based heavily on trends in these order parameters as the system size is increased, namely that at low chemical potential $\mu$ both order parameters decrease monotonically toward their disordered values, while at higher $\mu$ both order parameters increase monotonically away from their disordered values. This strongly suggests a nematic transition, which would be expected to be first order. The simulations in [8] became unreliable moving into the ordered regime, so in particular no direct evidence was given of a discontinuity of a first derivative of the free energy, namely volume fraction or energy density.

For this paper, which also reports a nematic transition in a model similar to [8] but on a square lattice, as in the original Flory model $[4,5]$, we were able to make reliable simulations well into the ordered regime, and simulated on a grid of values of $\mu$ and $\beta$. The main improvement over [8] is that now we are able to show discontinuities in volume fraction and energy density, the usual hallmarks of a first order transition, as well as discontinuities in corr and lay, which clarify the nematic nature of the transition. We feel that this is strong, direct evidence supporting the suggestion in [5] that the second order transition which they find at density 1 in the Flory model becomes first order at lower density when vacancies are included in the model. It still remains to reconcile this behavior with that found in [9] in their continuum model of confined loops.

\section{Acknowledgments}

We are grateful to E Katzav, S Deboeuf, A Boudaoud and N Menon for useful discussions. This research is supported in part by NSF grant DMS-0700120.

\section{References}

[1] Flory P J 1969 Statistical Mechanics of Chain Molecules (New York: Wiley)

[2] de Gennes P-G 1979 Scaling Concepts in Polymer Physics (Ithaca, NY: Cornell University Press)

[3] Flory P J 1956 Proc. R. Soc. A 23460

[4] Menon G I and Pandit R 1999 Phys. Rev. E 59787

[5] Jacobsen J L and Kondev J 2004 Phys. Rev. E 69066108

[6] Nagle J F 1974 Proc. R. Soc. A 337569

[7] Baumgartner A and Yoon D 1983 J. Chem. Phys. 79521

[8] Aristoff D and Radin C 2010 Europhys. Lett. 9156003

[9] Boué L and Katzav E 2007 Europhys. Lett. 8054002

[10] Janse van Rensburg E J 2009 J. Phys. A: Math. Theor. 42323001

[11] Oberdorf R et al 2006 Phys. Rev. E 74051801

[12] Jacobsen J L 2008 Phys. Rev. Lett. 100118102

[13] Adda-Bedia M et al 2010 J. Stat. Mech. P11027 